\title{
Stage III Uterine Corpus Cancer AJCC v8
}

National Cancer Institute

\section{Source}

National Cancer Institute. Stage III Uterine Corpus Cancer A/CC v8. NCI Thesaurus. Code C139806.

Stage III includes: T3, N0, M0. T3: Uterine corpus carcinoma or carcinosarcoma with tumor involving serosa, adnexa, vagina, or parametrium. N0: No regional lymph node metastasis. M0: No distant metastasis. (AJCC 8th Ed.) 